\title{
Gastric Ulcer
}

National Cancer Institute

\section{Source}

National Cancer Institute. Gastric Ulcer. NCI Thesaurus. Code C3388.

An ulcerated lesion in the mucosal surface of the stomach. It may progress to involve the deeper layers of the gastric wall. 